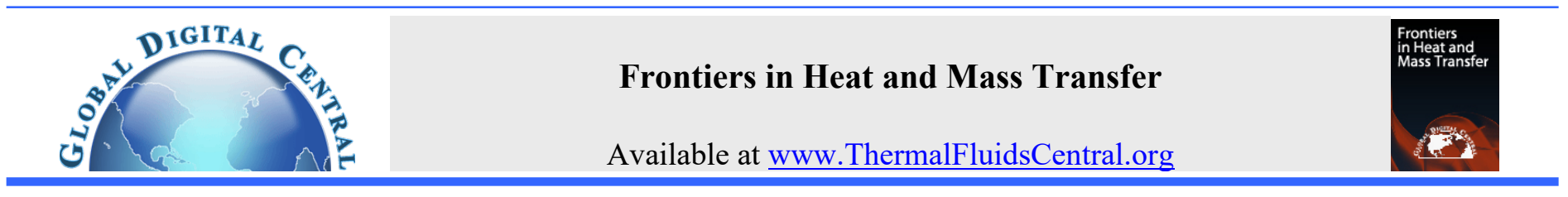

\title{
DOUBLE DIFFUSIVE NATURAL CONVECTION IN OPEN CAVITY UNDER THE SORET AND DUFOUR EFFECTS
}

\author{
Zhiyun Wang ${ }^{\dagger}$, Zixuan Zhou, Mo Yang \\ School of Energy and Power Engineering, University of Shanghai for Science and Technology, shanghai, 200093, China
}

\begin{abstract}
Double diffusive natural convection in an open cavity under the Soret and Dufour effect is simulated numerically. The influences of different Rayleigh numbers (range from $10^{3}$ to $10^{7}$ ), Lewis numbers (range from 0.5 to 8 ), buoyancy ratios (range from -5 to 5 ) and Soret and Dufour (range from 0 to 0.5 ) on the flow field, temperature and concentration distributions, as well as on the variation of the average Nusselt number and the average Sherwood number are investigated. The result shows that, when buoyancy ratios is -1 , the average Nusselt number and the average Sherwood number reaches the minimum, namely the heat and mass transfer in the cavity are the weakest. The increase of Lewis numbers under a fixed Rayleigh numbers would lead to decreased the average Nusselt number and gradually increased the average Sherwood number. When the value of Rayleigh numbers is small, the change of Lewis numbers has little effect on heat and mass transfer. Both the average Nusselt number and the average Sherwood number increase as Soret and Dufour increase. When Dufour and Soret simultaneously varies from 0.1 to 0.5 , compared with some cases, the Soret and Dufour effects are not considered, the average Nusselt number increases by $10.4 \%$ to $58.0 \%$ and the average Sherwood number increases by $3.4 \%$ to $13.1 \%$ respectively. In addition, the Soret and Dufour effect on the heat and mass transfer in open cavity is not obvious when Lewis numbers is 1.
\end{abstract}

Keywords: open cavity, double diffusion, natural convection, Soret and Dufour effects, numerical simulation.

\section{INTRODUCTION}

Natural convection plays an important role in engineering systems, and the related convection problems gradually arouse many researcher's attention. Generally, most of the fluids in natural convection systems are composed of two or more components. Prasad et al. (2018) numerically simulated the steady laminar natural convection and surface radiation in the two dimensional partially right side open square cavity filled with natural air $(\operatorname{Pr}=0.70)$ as the fluid medium. When the temperature and concentration gradients coexist, heat and mass transfer will occur simultaneously in the mixed fluid, and then the natural convection, that is, double diffusive natural convection occurs (Turner, 1974). At present, double diffusive convection has been widely used in industrial ventilation (Serrano-Arellano et al, 2013), crystal growth technology (Huppert and Sparks, 1984), metal mixture solidification (Ghenai et al, 2003), liquid film cooling (Zhang et al, 2006), solar energy absorber (Rahman et al, 2012).

A lot of experimental analysis and simulation studies have been carried out on the double diffusive convection problem. Most of the researchers focus on the analysis of the flow and heat transfer in a closed cavity. Bégheinet et al. (1992) numerically simulated the convection of heat and mass in a square cavity filled with air. Younis et al. (2007) studied the problem of double diffusive natural convection in closed cavity filled with liquid under different heating conditions and different fluid concentration. The result shows that the heat and mass transfer rate of the free surface is high, so the influence of the capping conditions can not be underestimated. Zhao et al. (2008) numerically simulated the double-diffusive convection flow of binary mixture in a porous enclosure. In recent decades, researchers increasingly paid more attention to the Soret and Dufour effects on double diffusive convection. The Soret effect refers to the mass transfer phenomenon caused by the non-uniform temperature field, and the Dufour effect refers to the heat transfer phenomenon caused by the non-uniform concentration field. The studies have shown that the Soret effect and the Dufour effect in double diffusive convection cannot be ignored in some cases. Chen et al. (2016) revealed the heat and mass transfer mechanism of dual diffusive natural convection in the range of nanofluids from laminar to turbulent by numerical method. Wang et al. (2016) conducted the study of double diffusive convection based on the bouyancy of hot solute and considered the Soret and Dufour effects. An unsteady numerical model based on thermosolutal buoyancies with Soret and Dufour effects for doublediffusive convection is developed. The flow field, temperature and concentration distributions for different aspect ratios, buoyancy ratios, Rayleigh numbers, Soret and Dufour coefficients are investigated systematically (Wang et al, 2014). Moorthy et al. (2012) analyzed the heat and mass transfer characteristics of natural convection embedded in vertical surface in saturated porous media under the effect of Soret and Dufour. Shi et al. (2006) numerically simulated the double diffusive convection in a cylindrical vessel, and gave a new feature of nonhorizontal stratification of temperature and concentration fields under the situation of double diffusion convection. Chen et al. (2004) established a mathematical model of natural convection with double buoyancy in porous media considering coupling diffusion effect, emphatically investigating the influences of Soret effect and Dufour effect on heat and mass transfer.

In addition to double diffusive convection in closed spaces, some researchers have studied the problem of double diffusive convection in open cavity. Mohamad et al. (2010) used the Lattice Boltzmann Method to study the problem of double diffusive convection in the open cavity when the buoyancy ratio $\mathrm{N}$ is negative. Arbin et al. (2014) studied the effects of double diffusive natural convection in the square cavity with 
top openings under local heating and solute. Kefayati (2016) studied the influences of Soret and Dufour effects on the double-diffusion natural convection problem of a power law non-Newtonian fluid in an open cavity under a horizontal magnetic field using FDLBM (Finite Difference Lattice Boltzmann Method).

However, for open systems, the double diffusive natural convection problem of normal Newtonian fluid in a free space considering the Soret effect and the Dufour effect has not been reported. Nawazd et al. (2012) pointed out that Dufour and Sorret effects are particularly obvious when the very light molecular weight gases $\left(\mathrm{H}_{2}, \mathrm{He}\right)$ and medium molecular weight gases are mixed for heat and mass transfer. In addition, Shi et al. (2001) studied the heat and moisture transfer properties of textiles, finding that the influence of Soret effect and Dufour effect on the simultaneous heat and moisture transfer in wet air was much greater than that of the heat and moisture cross effect in infinitely miscible non-polar mixed gases. In that case, the effect of the cross-effect of heat and moisture can not be ignored. This paper is aimed at this deficiency. The numerical simulation of the natural convection in the open cavity under the Soret and Dufour effect when the mixture of two gases is used as the medium. The effects of different parameters on the thermosolutal diffusion in the open cavity are investigated. In the scope of this study, without comparing Soret effect and the Dufour effect, the maximum difference between Soret number and Dufour number is $58.0 \%$ and $13.1 \%$.

\section{Physical Model and Numerical Method}

\subsection{Physics and Mathematical Model}

The domain under analysis is, as sketched in Fig. 1, a square, twodimensional open cavity in a gravitational field. The upper and lower walls of the square cavity are adiabatic, the right side is an open interface to the ambience, and the left wall is kept high temperature $T_{h}$ and high concentration $c_{h}$. The surrounding fluid interacting with the aperture is fixed to an ambient temperature and concentration $\left(T_{l}\right.$ and $\left.c_{l}\right)$ and has the same material properties as the fluid inside the cavity. The initial temperature and concentration of the fluid in the cavity are the same as their counterpart of surrounding area, assuming $T_{l}<T_{h}$ and $c_{l}<c_{h}$.

The fluid filling in the cavity is air $(\mathrm{Pr}=0.71)$ mixed with another species. The fluid mixture is assumed to be Newtonian, the fluid flow is considered to be laminar. And there are no chemical reactions, viscous dissipation and thermal radiation in the system. The properties of the mixture are assumed to be constant, except for the density in the buoyancy force term in the momentum equations, according to the Boussinesq approximation.

The mixture density in the buoyancy term is taken as a function of both the temperature and concentration fields and can be obtained from a given reference value $\rho_{\mathrm{r}}=\rho\left(T_{\mathrm{r}}, c_{\mathrm{r}}\right)$ as:

$\rho=\rho_{\mathrm{r}}\left[1-\beta_{T}\left(T-T_{r}\right)-\beta_{c}\left(c-c_{r}\right)\right]$

Where $\beta_{\mathrm{T}}=-(\partial \rho / \partial T)_{\mathrm{c}} / \rho_{\mathrm{r}}$ and $\beta_{\mathrm{c}}=-(\partial \rho / \partial c)_{\mathrm{T}} / \rho_{\mathrm{r}}$ are thermal expansion coefficient and mass expansion coefficient respectively, which are assumed to be constant.

\subsection{Governing Equations}

The transient dimensionless conservation equations governing the transport of mass, momentum, energy, and concentration in the primitive variables are expressed as:

$$
\begin{aligned}
& \frac{\partial U}{\partial X}+\frac{\partial U}{\partial Y}=0 \\
& \frac{\partial U}{\partial \tau}+U \frac{\partial U}{\partial X}+V \frac{\partial U}{\partial Y}=-\frac{\partial P}{\partial X}+\operatorname{Pr}\left(\frac{\partial^{2} U}{\partial X^{2}}+\frac{\partial^{2} U}{\partial Y^{2}}\right)
\end{aligned}
$$

$$
\begin{aligned}
& \frac{\partial V}{\partial \tau}+U \frac{\partial V}{\partial X}+V \frac{\partial V}{\partial Y} \\
& =-\frac{\partial P}{\partial Y}+\operatorname{Pr}\left(\frac{\partial^{2} V}{\partial X^{2}}+\frac{\partial^{2} V}{\partial Y^{2}}\right)+R a \cdot \operatorname{Pr}(\Theta+N C)
\end{aligned}
$$

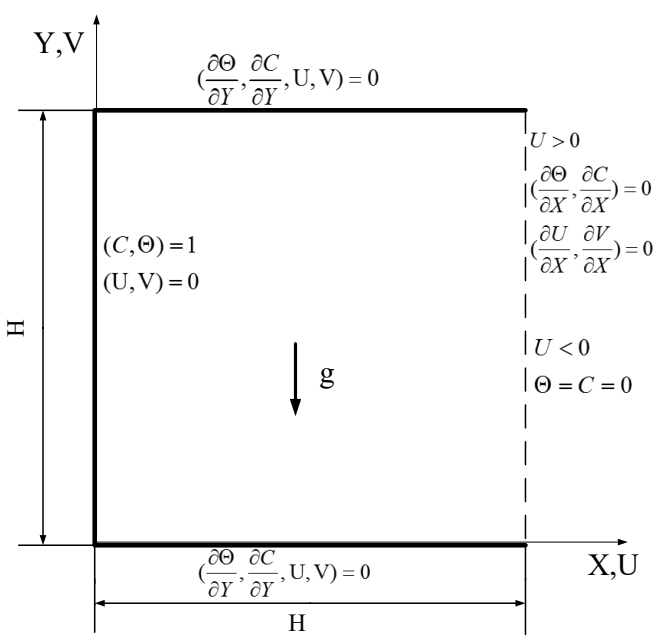

Fig. 1 Physical model

$$
\begin{aligned}
& \frac{\partial \Theta}{\partial \tau}+U \frac{\partial \Theta}{\partial X}+V \frac{\partial \Theta}{\partial Y}=\left(\frac{\partial^{2} \Theta}{\partial X^{2}}+\frac{\partial^{2} \Theta}{\partial Y^{2}}\right)+D_{f}\left(\frac{\partial^{2} C}{\partial X^{2}}+\frac{\partial^{2} C}{\partial Y^{2}}\right) \\
& \frac{\partial C}{\partial \tau}+U \frac{\partial C}{\partial X}+V \frac{\partial C}{\partial Y}=\frac{1}{L e}\left[\left(\frac{\partial^{2} C}{\partial X^{2}}+\frac{\partial^{2} C}{\partial Y^{2}}\right)+S_{r}\left(\frac{\partial^{2} \Theta}{\partial X^{2}}+\frac{\partial^{2} \Theta}{\partial Y^{2}}\right)\right]
\end{aligned}
$$

The above equations were nondimensionalized by defining:

$$
\begin{aligned}
& X=\frac{x}{H}, Y=\frac{y}{H}, U=\frac{u}{U_{R}}, V=\frac{v}{U_{R}}, \tau=\frac{U_{R} t}{H}, \\
& \Theta=\frac{T-T_{\infty}}{T_{h}-T_{\infty}}, C=\frac{c-c_{\infty}}{c_{h}-c_{\infty}}, P=\frac{p}{\rho U_{R}^{2}}, \mathrm{U}_{R}=\frac{\alpha}{H}
\end{aligned}
$$

The above problem defined in Eqs. (2)-(6) is determined by the following six dimensionless parameters:

Rayleigh number $R a=\frac{g \beta_{T}\left(T_{h}-T_{\infty}\right) H^{3}}{v \alpha}$, Prandtl number $\operatorname{Pr}=\frac{v}{\alpha}$, Lewis number $L e=\frac{\alpha}{D}$, buoyancy ratio $N=\frac{\beta_{c}\left(c_{h}-c_{\infty}\right)}{\beta_{T}\left(T_{h}-T_{\infty}\right)}$, Dufour coefficients $D_{f}=\frac{\kappa_{T C}\left(C_{h}-C_{l}\right)}{\alpha\left(T_{h}-T_{l}\right)}$, Soret coefficients $S_{r}=\frac{\kappa_{C T}\left(T_{h}-T_{l}\right)}{D\left(C_{h}-C_{l}\right)}$.

Where $g$ is gravitational acceleration, $v$ is kinetic viscosity, $\alpha$ is thermal diffusivity, $D$ is solutal diffusivity, $\kappa_{\mathrm{CT}}$ and $\kappa_{\mathrm{TC}}$ are Soret coefficient and Soret coefficient respectively.

\subsection{Initial and boundary conditions}

Equations (2)-(6) are subject to the following initial conditions: $\tau=0, U=V=0, \Theta=0, C=0$

The velocity, temperature, and concentration boundary conditions for the open cavity are specified as follows (Fig. 1):

At $Y=1,0 \leq X \leq 1, U=V=0, \frac{\partial \Theta}{\partial Y}=\frac{\partial C}{\partial Y}=0$
At $Y=0,0 \leq X \leq 1, U=V=0, \frac{\partial \Theta}{\partial Y}=\frac{\partial C}{\partial Y}=0$
At $X=0,0 \leq Y \leq 1, U=V=0, \Theta=1, C=1$ 


$$
\text { At } X=1,0 \leq Y \leq 1, \begin{cases}\frac{\partial U}{\partial X}=\frac{\partial U}{\partial Y}=0, \frac{\partial \Theta}{\partial X}=\frac{\partial C}{\partial X}=0, & \text { if } U>0 \\ \frac{\partial U}{\partial X}=\frac{\partial U}{\partial Y}=0, \Theta=C=0, & \text { if } U<0\end{cases}
$$

The local Nusselt number Nuy and local Sherwood number Shy are defined as:

$$
\begin{aligned}
& \mathrm{Nu}_{\mathrm{Y}}=-\left.\frac{\partial \Theta}{\partial X}\right|_{X=0}-D_{f}-\left.\frac{\partial C}{\partial X}\right|_{X=0} \\
& \mathrm{Sh}_{\mathrm{Y}}=-\left.\frac{\partial C}{\partial X}\right|_{X=0}-\left.S_{r} \frac{\partial \Theta}{\partial X}\right|_{X=0}
\end{aligned}
$$

The average Nusselt number $\mathrm{Nu}$ and average Sherwood number $\mathrm{Sh}$ are the average rate of dimensionless temperature change in the normal direction of the hot wall.

$$
\begin{aligned}
& \mathrm{Nu}=-\int_{0}^{1} N u_{Y} d Y \\
& \mathrm{Sh}=-\int_{0}^{1} S h_{Y} d Y
\end{aligned}
$$

\section{NUMERICAL SOLUTION AND CODE VALIDATION}

The control volume method based on the original variables is used to calculate the coupling of pressure and velocity in the governing equation by SIMPLE algorithm. The QUICK scheme is adopted for discretization of the governing equations, and the boundary condition at the exit of the open cavity adopts local unidirectional processing (TONG et al, 2009).

After the grid assessment of $100 \times 100,125 \times 125,150 \times 150,175 \times 175$ and $200 \times 200$, the mean grid distribution of $150(\mathrm{x}) \times 150(\mathrm{y})$ was selected, and the deviation between the results and those of the densest grid was less than $0.30 \% .0 .1,0.01$ and 0.001 were selected for the dimensionless time step, and 0.001 was selected after comparison, with the relative minimum time step deviation less than $0.0085 \%$.

The problem of natural convection of Ren and Chan (2016) in a vertical square cavity was numerically calculated to validate the reliability of the algorithm in this paper, and the results were compared. As shown in table 1, the maximum deviation was $1.34 \%$. Due to the unpredictability of the results' time-sensitiveness, the non-steady-state algorithm is used in this paper. It turns out that the calculated results are all steady state, and the average $\overline{\mathrm{Nu}}$ and $\overline{\mathrm{Sh}}$ under the imposed conditions fluctuate no more than $10^{-5}$ over time.

Table 1 Comparison with reference results

\begin{tabular}{|c|c|c|c|}
\hline \multirow{2}{*}{$\mathrm{N}$} & \multicolumn{3}{|c|}{$\overline{\mathrm{Nu}}$} \\
\cline { 2 - 4 } & $\begin{array}{c}\text { reference } \\
\text { results }\end{array}$ & $\begin{array}{c}\text { This } \\
\text { work }\end{array}$ & deviation \\
\hline-0.001 & 4.7201 & 4.7182 & $0.04 \%$ \\
\hline-0.1 & 4.6405 & 4.6385 & $0.04 \%$ \\
\hline-2 & 2.9153 & 2.9158 & $0.02 \%$ \\
\hline-10 & 7.0813 & 7.0884 & $0.10 \%$ \\
\hline-100 & 13.6000 & 13.7393 & $1.02 \%$ \\
\hline \multicolumn{3}{|c|}{$\overline{\text { Sh }}$} \\
\hline \multirow{2}{*}{$\mathrm{N}$} & $\begin{array}{c}\text { reference } \\
\text { results }\end{array}$ & $\begin{array}{c}\text { This } \\
\text { work }\end{array}$ \\
\hline-0.001 & 6.1006 & 6.0930 & deviation \\
\hline-0.1 & 5.9844 & 5.9769 & $0.13 \%$ \\
\hline-2 & 4.6993 & 4.6936 & $0.12 \%$ \\
\hline-10 & 9.9517 & 9.9664 & $0.15 \%$ \\
\hline-100 & 18.6850 & 18.9345 & $1.34 \%$ \\
\hline
\end{tabular}

\section{NUMERICAL CALCULATION AND ANALYSIS}

\subsection{Effect of buoyancy ratio $\mathrm{N}$ on flow and heat transfer in open cavity}

In this paper, the double diffusive natural convection of the organic compound propanol and air mixture in the open square cavity is numerically simulated first, considering the Soret and Dufour effects. The relevant parameters in the numerical calculation are $\operatorname{Pr}=0.71$, $\mathrm{Le}=1.641, \mathrm{Sr}=\mathrm{Df}=0.1$. Fig. 2 shows the average Nusselt number $\overline{\mathrm{Nu}}$ and the average Sherwood number $\overline{\mathrm{Sh}}$ vary as a function of the buoyancy ratio $\mathrm{N}$ under different $\mathrm{Ra}$. The range of buoyancy ratio $\mathrm{N}$ is $-5 \leq \mathrm{N} \leq 5$. It can be seen from Fig. 2 that $\overline{\mathrm{Nu}}$ and $\overline{\mathrm{Sh}}$ are axisymmetric with $\mathrm{N}=-1$ as the axis of symmetry. With the change of $\mathrm{N}$, the thermal mass diffusion of the entire cavity is gradually enhanced and weakened. When $\mathrm{N}=-1$, thermal buoyancy and solute concentration buoyancy in opposite directions. Theoretically, the two buoyancy forces are balanced with each other, and heat and mass transfer are carried out by pure diffusion. Therefore, heat and mass transfer within the cavity are the weakest of all, both $\overline{\mathrm{Nu}}$ and $\overline{\mathrm{Sh}}$ reach their minimum. When the value of $\mathrm{N}$ is far from -1 , the thermal buoyancy or solute buoyancy force increases, and the flow is strengthened, thereby gradually enhancing the heat transfer and mass transfer effects.
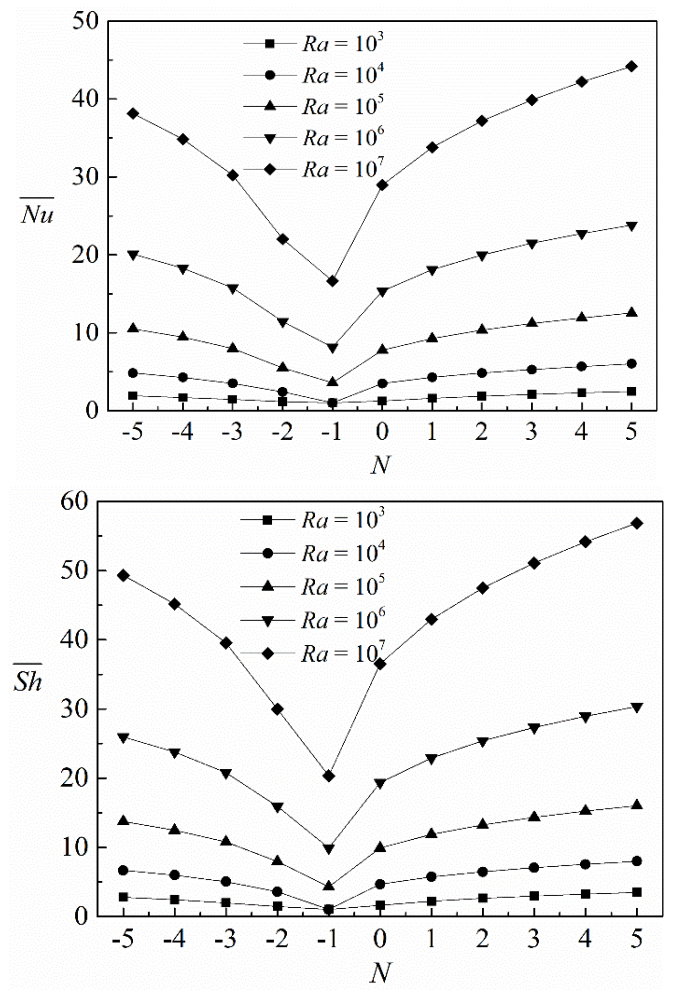

Fig. 2 Average Nusselt and Sherwood number for various Ra with different buoyancy ratio $\mathrm{N}$

Figure 3 shows the temperature field, concentration field, and flow field distribution in the open cavity when $\mathrm{Ra}=10^{5}$ and $\mathrm{N}$ are -5 and 4 , respectively. As can be seen from Fig. 3, when $\mathrm{N}=-5$ and $\mathrm{N}=4$, the temperature field, concentration field and flow field in the open cavity are approximately symmetric distributed. When $\mathrm{N}=-5$, the downward solute buoyancy force is greater than the upward thermal buoyancy force, the fluid flows along the wall surface in the counterclockwise direction, and there is a vortex formation in the lower left corner of the open cavity, when $\mathrm{N}=4$, as shown in Fig. 3, the temperature field, concentration field, and flow field are opposite to those of $\mathrm{N}=-5$. Combined with the analysis results of Fig. 2 above, it is worth noting that when $\mathrm{N}=-1$, the buoyancy 
of the fluids on both sides is equal and the direction is opposite. When other parameters are the same, the fluid flows from bottom to top when $\mathrm{N}>-1$, and the fluid flows from top to bottom when $\mathrm{N}<-1$.
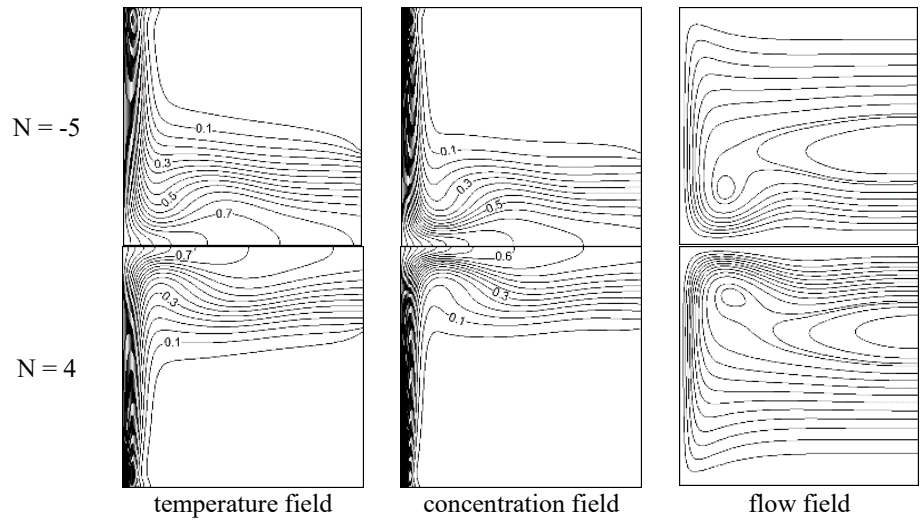

Fig. 3 Distributions of temperature field, concentration field and flow field for $\mathrm{N}=-5$ and $\mathrm{N}=4\left(\mathrm{Ra}=10^{5}\right)$

\subsection{Effect of Lewis number Le on flow and heat transfer in open cavity}

In order to obtain the effect of the Lewis number Le on the flow and heat transfer in the open cavity, the following parameters are kept constant: $\mathrm{Pr}=0.71, \mathrm{~N}=2, \mathrm{Sr}=\mathrm{Df}=0.1$.
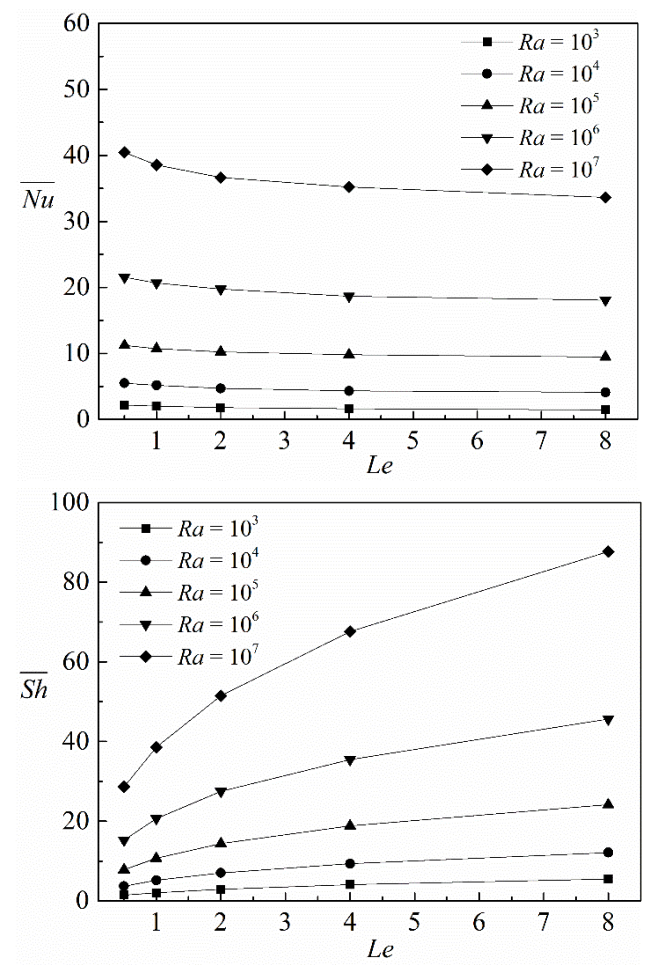

Fig. 4 Average Nusselt and Sherwood number for various Ra with different Lewis numbers Le

Figure 4 shows the effect of different $\operatorname{Le}(0.5 \leq \mathrm{Le} \leq 8)$ on the average Nusselt number $\overline{\mathrm{Nu}}$ and the average Sherwood number $\overline{\mathrm{Sh}}$ in the open cavity when $\mathrm{Ra}=10^{3} \sim 10^{7}$. It should be pointed out that, at this time, the values of $\mathrm{Sr}$ and $\mathrm{Df}$ are as small as 0.1, and the Soret effect and Dufour effect are small. Heat and mass transfer mainly depend on the temperature gradient and concentration gradient at the left wall surface. It can be seen from Fig. 4 that when Le is constant, the heat and mass transfer effect in the open cavity is enhanced with the increase of Ra. Changing Le doesn't influence $\overline{\mathrm{Nu}}, \overline{\mathrm{Sh}}$ very much when $\mathrm{Ra}$ is small. When $\mathrm{Ra}$ is constant, as Le increases, $\overline{\mathrm{Nu}}$ gradually decreases and $\overline{\mathrm{Sh}}$ gradually increases. It is known that mass transfer in the cavity is enhanced and heat transfer is weakened. The following is a combination of temperature field, concentration field and flow field distribution to analyze the heat and mass transfer in the open cavity.

Figure 5 shows the temperature field, concentration field, and flow field distribution in the open cavity when $\mathrm{Ra}=10^{5}$. Le approximately expresses the relative thickness ratio of thermal boundary layer and concentration boundary layer. When Le increases, the concentration boundary layer becomes thinner and the thermal boundary layer becomes thicker, which results in the enhancement of mass transfer and the decrease of heat transfer. When Ra does not change, the buoyancy caused by temperature difference does not change, and because $\mathrm{N}=2$ in these cases, the buoyancy caused by concentration difference increases when mass transfer increases, resulting in slightly enhanced advection. With the increase of Le, the comprehensive effect of diffusion and advection decreases slightly in heat transfer and increases significantly in mass transfer. At the same time, the stream lines near the upper left corner and the upper wall become distorted due to the slightly enhanced fluid flow.
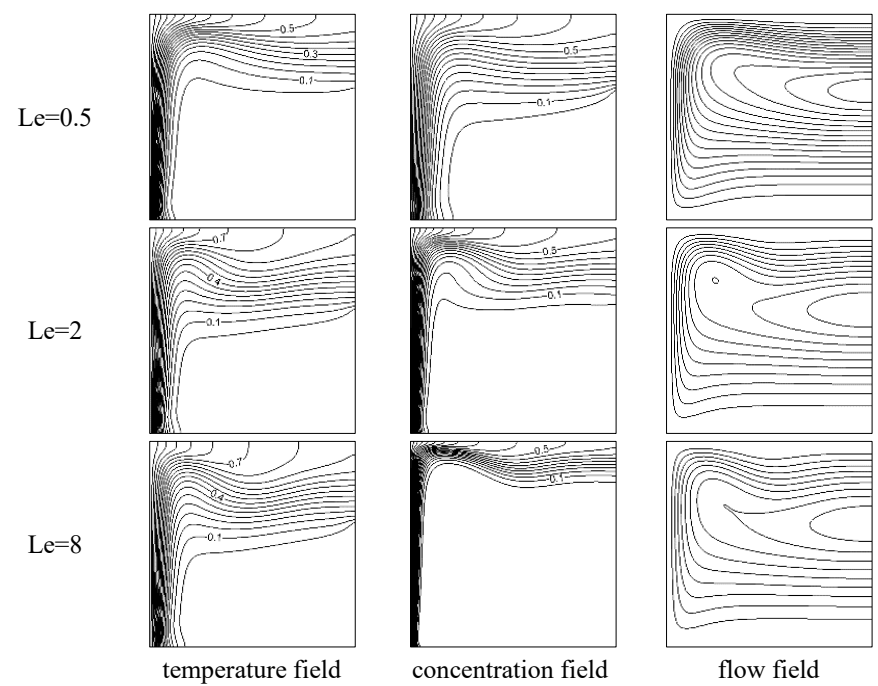

Fig. 5 Distributions of temperature field, concentration field and flow field for $\mathrm{Le}=0.5,2,8$

$\left(\mathrm{Ra}=10^{5}, \mathrm{Pr}=0.71, \mathrm{~N}=2, \mathrm{Sr}=\mathrm{Df}=0.1\right)$

Figure 6 shows the local Nusselt number $\mathrm{Nu}$ and the Sherwood number Sh distribution along the left wall at different Le under $\mathrm{Ra}=10^{5}$, $\operatorname{Pr}=0.71, \mathrm{~N}=2, \mathrm{Sr}=\mathrm{Df}=0.1$. It can be seen that the heat transfer and mass transfer of the wall is gradually weakened from bottom to top. Nu and Sh at the top of the hot wall were the smallest, and $\mathrm{Nu}$ did not change much within the range of $Y>0.8$, indicating that the heat transfer and mass transfer at the top of the hot wall were relatively weak and did not change significantly with Le. The maximum value of $\mathrm{Nu}$ and $\mathrm{Sh}$ appeared near the bottom of the hot wall surface, where the double diffusion heat transfer and the mass transfer effect reached the maximum in the open cavity. This is because that the temperature gradient and concentration gradient near the high temperature and high concentration wall on the left side are the largest, and the thermal boundary layer and the concentration boundary layer are the thinnest, which is consistent with the results shown in the temperature and concentration field diagram of Fig. 5. According to discussion in section 4.1, when $\mathrm{N}=2$, the fluid flows from bottom to top along the left wall, and in the clockwise direction in the cavity. On lower part of the heating wall, a direct impact of fresh air entering from the outside was received, resulting in a thin thermal boundary layer and its counterpart of concentration. Then the two boundary layers gradually get thicken as the fluid flows upward along 
the left wall. Therefore, the heat and mass transfer effect near the bottom of the left wall surface is the strongest. For the region near the left wall of $\mathrm{Y}<0.8$, NuY and Shy vary the same as $\overline{\mathrm{Nu}}$ and $\overline{\mathrm{Sh}}$ for the same reason when Le changes. As for the region near the left wall of $Y>0.8$, it can be found from Fig. 5 that the isotherms are nearly evenly distributed with different Le, while isoconcentration lines are squeezed toward the lefttop corner by higher Le. And the thermal and concentration boundary layer are thicker than other place. It indicates that the heat transfer in this region are conduction dominated. Consequently, the $\mathrm{Nu}$ is not sensitive to Le while the Shy get increase with higher Le.
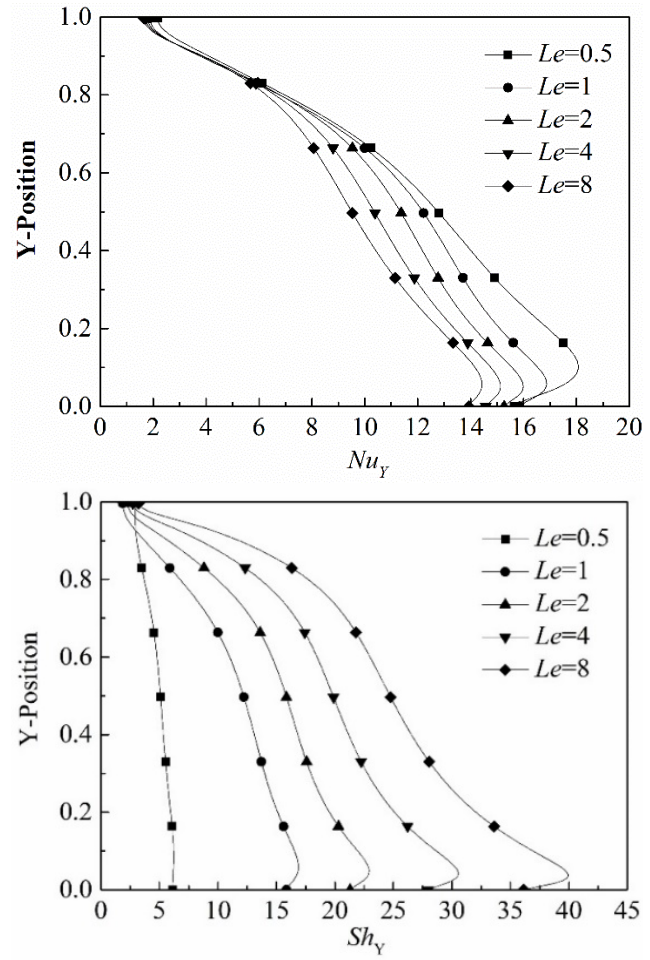

Fig. 6 Local Nusselt and Sherwood number along the hot wall with different Lewis numbers $\left(\mathrm{Ra}=10^{5}, \mathrm{Pr}=0.71, \mathrm{~N}=2, \mathrm{Sr}=\mathrm{Df}=0.1\right)$

\subsection{Effect of Soret and Dufour Coefficients on Flow and Heat Transfer in Open Cavity}

The Soret effect (also known as the thermal additive diffusion effect) can take a positive or negative value. Positive values represent the presence of temperature gradients that produce mass transfer from high to low temperatures, and negative values represent the presence of temperature gradients that produce mass transfer from low to high temperatures. The Dufour effect (also known as diffusion plus thermal effect) is similar to the above effect. Considering the Soret and Dufour effects, the simultaneous heat and mass transfer process will have a direct interaction, resulting in a so-called cross-coupling diffusion effect. The Soret and Dufour effects are concentrated in the governing equations in the additional source term. From the governing equations (5) and (6), temperature and concentration interactions can also be seen, and heat and mass transfer interact with each other.

Figure 7 shows the temperature field, concentration field, and flow field distribution in the open cavity when $\mathrm{Ra}=10^{5}, \operatorname{Pr}=0.71, \mathrm{Le}=1, \mathrm{~N}=2$, $\mathrm{Sr}=\mathrm{Df}=0.1,0.3,0.5$. It can be seen that the isothermal andconcentration linesare denser on the left wall surface. And the temperature distribution is basically identical with the concentration distribution in the cavity, which is consistent with $\mathrm{Le}=1$. When $\mathrm{Le}=1$, thermal diffusivity and solutal diffusivity are the same. With the change of $\mathrm{Sr}$ and $\mathrm{Df}$ number, the streamline changes in the upper left corner, and there is a slight trend that the isothermal and concentration lines became denser. It lead to that when $\mathrm{Le}=1$, the enhancement of Soret and Dufour effect has a little enhancement effect on heat and mass transfer in the open square cavity, but not much.

Figure 8 shows the variation of $\overline{\mathrm{Nu}}$ and $\overline{\mathrm{Sh}}$ in the open cavity increases $\mathrm{Ra}$ increases at different $\mathrm{Sr}$ and $\mathrm{Df}$ while other parameters are kept as constant as $\operatorname{Pr}=0.71, \mathrm{Le}=1, \mathrm{~N}=2$. It can be drawn from the figure that $\overline{\mathrm{Nu}}$ and $\overline{\mathrm{Sh}}$ are increasing as Soret and Dufour effects intensify. $\overline{\mathrm{Nu}}$ increased by $7.0 \%$ to $58.0 \%, \overline{\mathrm{Sh}}$ increased by $2.3 \%$ to $13.1 \%$, when $\mathrm{Sr}$ and Df increased from 0.1 to 0.5 , compared with the case without Soret and Dufour effects (i.e. $\mathrm{Sr}=\mathrm{Df}=0$ ). It implies that the influence of the Soret and Dufour effects on the double-diffusion convective heat and mass transfer in the open cavity cannot be ignored.

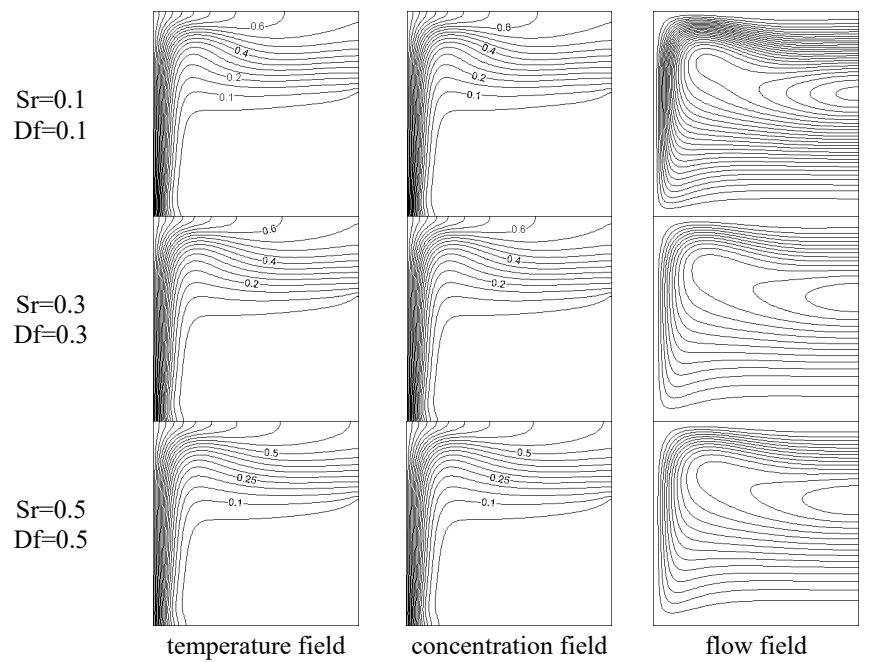

Fig. 7 Distributions of temperature field, concentration field, flow field, $E_{S}$ and $E_{D}$ with $\mathrm{Sr}$ and $\mathrm{Df}$ increasing $\left(\mathrm{Ra}=10^{5}, \mathrm{Le}=1, \mathrm{~N}=2\right)$
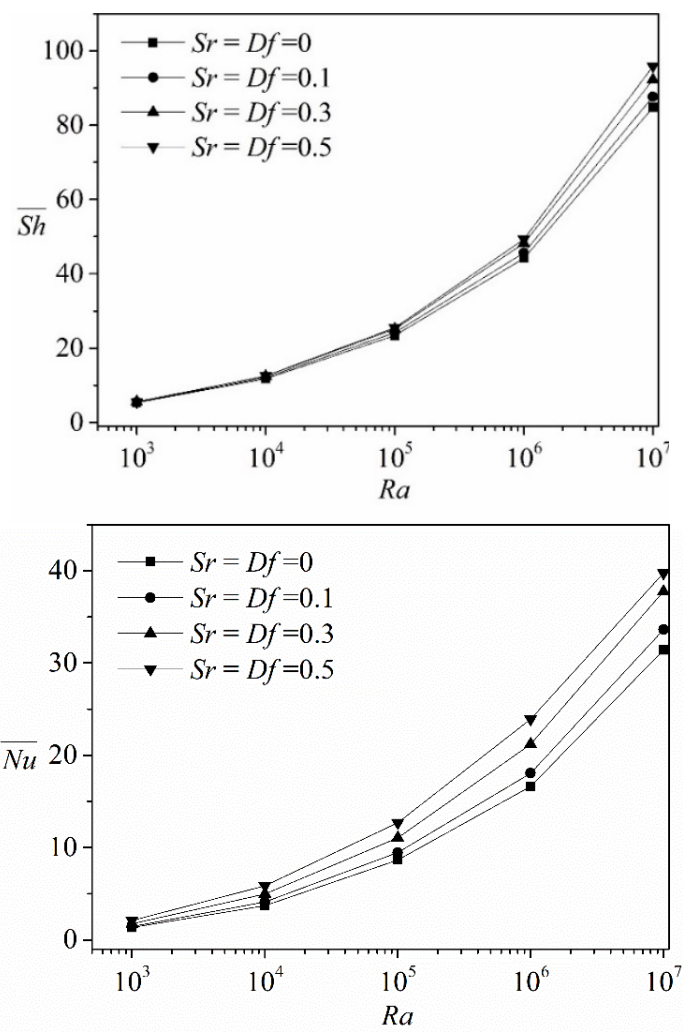

Fig. 8 Average Nusselt and Sherwood number for $\mathrm{Sr}=\mathrm{Df}=0,0.1,0.3$ and 0.5 , respectively 


\section{CONCLUSIONS}

In this paper, the numerical simulation of the double diffusive natural convection in a square open cavity under the effect of Soret and Dufour is carried out. The influence of $\mathrm{N}, \mathrm{Ra}, \mathrm{Le}, \mathrm{Sr}$ and $\mathrm{Df}$ on heat and mass transfer in the open cavity was analyzed, and the following conclusions were drawn:

(1) When $\mathrm{N}=-1$, the average Nusselt number $\overline{\mathrm{Nu}}$ and the average Sherwood number $\overline{\mathrm{Sh}}$ reach the minimum value, and the heat transfer and mass transfer effects in the cavity are the weakest. With the larger absolute value of $\mathrm{N}$, the heat transfer and mass transfer effects gradually enhanced.

(2) When $\mathrm{Ra}$ is small, changing Le has little effect on $\overline{\mathrm{Nu}}$ and $\overline{\mathrm{Sh}}$. When Le is constant, as Ra increases, $\overline{\mathrm{Nu}}$ and $\overline{\mathrm{Sh}}$ gradually increase. For a fixed $\mathrm{Ra}, \overline{\mathrm{Nu}}$ gradually decreases and $\overline{\mathrm{Sh}}$ gradually increases with the increase of Le.

(3) When $L e=1$, the enhancement of the Soret and Dufour effects has no obvious effect on the heat and mass transfer in the open cavity. Within the range of parameters considered in this paper, with the increase of Soret and Dufour effects, the maximum difference of $\overline{\mathrm{Nu}}$ and $\overline{\mathrm{Sh}}$ is $58.0 \%$ and $13.1 \%$ respectively $(\mathrm{Sr}=\mathrm{Df}=0.5$ without being compared with $\mathrm{Sr}=\mathrm{Df}=0)$. Therefore, it is meaningful to consider the Soret and Dufour effects on the double-diffusion convective heat and mass transfer in an open cavity.

\section{ACKNOWLEDGEMENTS}

The financial support from National Natural Science Foundation of China (Key Program 51736007) is gratefully acknowledged.

\section{NOMENCLATURE}

C

$D$

$g$

$H$

Le

$N$

$\mathrm{Nu}$

$p$

$P$
$P r$

$\operatorname{Pr}$

$D f$

$\mathrm{Sr}$

$t$

$T$

$\operatorname{Tr}$

$u, v$

$U, V$

$U_{R}$

$x, y$

$X, Y$

Greek Symbols

$\alpha$

$\beta c$

conce

temperature change, $K^{-}$

concentration, $\mathrm{kg} / \mathrm{m}^{3}$

dimensionless concentration, dimensionless

mass diffusivity, $\mathrm{m}^{2} / \mathrm{s}$

gravitational acceleration, $\mathrm{m} / \mathrm{s}^{2}$

height or length of the square cavity, $m$

Lewis number, dimensionless

buoyancy ratio, dimensionless

Nussle number, dimensionless

pressure, $\mathrm{N} / \mathrm{m}^{2}$

dimensionless pressure, dimensionless

Prandtl number, dimensionless

Rayleigh number, dimensionless

Dufour number, dimensionless

Soret number, dimensionless

time, $s$

temperature, $K$

reference temperature, $K$

velocities, $\mathrm{m} / \mathrm{s}$

dimensionless velocities, dimensionless

reference velocity, $\mathrm{m} / \mathrm{s}$

coordinates, $m$

dimensionless coordinates, dimensionless

thermal diffusivity, $\mathrm{m}^{2} / \mathrm{s}$

coefficient of volumetric expansion due to

$\Theta$

$\rho$

$\tau$

$v$

$\begin{array}{ll}\text { Subscripts } & \\ c & \text { referring to mass transfer } \\ h & \text { higher level } \\ T & \text { referring to heat transfer } \\ Y & \text { local value } \\ \infty & \text { referring to ambient } \\ 0 & \text { reference value } \\ \text { Superscripts } & \\ n & \text { time step }\end{array}$

\section{REFERENCES}

Arbin, N. \& Hashim, I, 2014, "Partial heating and partial salting on double-diffusive convection in an open cavity," Ukm Fst Postgraduate Colloquium: Universiti Kebangsaan Malaysia, Faculty of Science \& Technology Postgraduate Colloquium. American Institute of Physics, 891-897.

https://doi.org/10.1063/1.4895320

Béghein C, Haghighat F, Allard F, 1992, "Numerical study of doublediffusive natural convection in a square cavity," International Journal of Heat \& Mass Transfer, 35(4), 833-846.

https://doi.org/10.1016/0017-9310(92)90251-M

Chen Bao-min, Wang Bu-xuan, Zhang Li-qiang, 2004, "Study on coupling diffusion effect in heat and mass transfer in porous media," Journal of Engineering Thermophysics, 25(s1), 125-128.

Chen S, Yang B, Luo K H, Xiong X P, \& Zheng C G, 2016, "Double diffusion natural convection in a square cavity filled with nanofluid," International Journal of Heat \& Mass Transfer, 95, 1070-1083. https://doi.org/10.1016/j.ijheatmasstransfer.2015.12.069

Elarabawy, H. A. M, 2009, "Soret and dufour effects on natural convection flow past a vertical surface in a porous medium with variable surface temperature," Journal of Mathematics \& Statistics, 5(3), 190-198. http://dx.doi.org/10.1155/2012/634806

Ghenai C, Mudunuri A, Lin C X, \& Ebadian M A, 2003, "Doublediffusive convection during solidification of a metal analog system $(\mathrm{NH}$ $4 \mathrm{Cl}-\mathrm{H} 2 \mathrm{O}$ ) in a differentially heated cavity," Experimental Thermal \& Fluid Science, 28(1), 23-35. https://doi.org/10.1016/S0894-1777(03)00089-X

H E Huppert, and, R S J Sparks, 1984, "Double-Diffusive Convection Due to Crystallization in Magmas," Annual Review of Earth and Planetary Sciences, 12(1), 11-37.

https://doi.org/10.1146/annurev.ea.12.050184.000303

Jin wang, Mo Yang, Yuwen Zhang, 2014, "Onset of double-diffusive convection in horizontal cavity with Soret and Dufour effects," International Journal of Heat \& Mass Transfer, 78, 1023-1031. https://doi.org/10.1016/j.ijheatmasstransfer.2014.07.064

Kefayati G H R, 2016, "Simulation of double diffusive MHD (magnetohydrodynamic) natural convection and entropy generation in an open cavity filled with power-law fluids in the presence of Soret and Dufour effects (Part I: Study of fluid flow, heat and mass transfer)," Energy, 107, 889-916. https://doi.org/10.1016/j.energy.2016.05.044

Mohamad A A, Bennacer R, El-Ganaoui M, 2010, "Double dispersion, natural convection in an open end cavity simulation via Lattice Boltzmann Method," International Journal of Thermal Sciences, 49(10), 1944-1953. https://doi.org/10.1016/j.ijthermalsci.2010.05.022 
Nawaz M, Hayat T, Alsaedi A, 2012, "Dufour and Soret effects on MHD flow of viscous fluid between radially stretching sheets in porous medium," Applied Mathematics and Mechanics, 33(11), 1403-1418. https://doi.org/10.1007/s10483-012-1632-6

Rahman M M, Öztop H F, Ahsan A, Kalam M A, \& Varol Y, 2012 "Double-diffusive natural convection in a triangular solar collector," International Communications in Heat \& Mass Transfer, 39(2), 264-269. https://doi.org/10.1016/j.icheatmasstransfer.2011.11.008

Ravi Shankar Prasad, S. N. Singh, Amit Kumar Gupta, 2018, "Coupled laminar natural convection and surface radiation in partially right side open cavities," Frontiers in Heat and Mass Transfer, 11, 28. http://doi.org/10.5098/hmt.11.28

Ren Q, Chan C L, 2016, "Numerical study of double-diffusive convection in a vertical cavity with Soret and Dufour effects by lattice Boltzmann method on GPU," International Journal of Heat \& Mass Transfer, 93, 538-553.

https://doi.org/10.1016/j.ijheatmasstransfer.2015.10.031

Serrano-Arellano J, Xamán J, Álvarez G, 2013, “Optimum ventilation based on the ventilation effectiveness for temperature and $\mathrm{CO} 2$, distribution in ventilated cavities," International Journal of Heat \&Mass Transfer, 62(1), 9-21. https://doi.org/10.1016/j.ijheatmasstransfer.2013.02.051

Shi K F, Lu W Q, 2006, "The stratification phenomena in the thermal and solutal fields of double-diffusive convection in a circular cylinder," Science Bulletin, 51(6), 747-754. https://doi.org/10.1007/s11434-006-0747-7
M.W.Shi, M.Yao, 2001, "The cross effect of thermal and water vapoure transfer for textiles," Journal of Northwest Institute of Textile Science and Technology, 15(2), 29-32.

TONG Yan, SHI Ming-heng, GONG Yan-feng, 2009, "Studying on Air Flow and Pollution of Natural Ventilation Tunnel with Shafts," Building Energy and Environment, 28(4), 18-22.

Turner J S, 1974, "Double-Diffusive Phenomena," Annual Review of Fluid Mechanics, 6(6), 37-55.

https://doi.org/10.1146/annurev.fl.06.010174.000345

Wang J, Yang M, He Y L, et al, 2016, "Oscillatory double-diffusive convection in a horizontal cavity with Soret and Dufour effects," International Journal of Thermal Sciences, 106(7), 57-69.

https://doi.org/10.1016/j.ijthermalsci.2016.03.012

Younis L B, Mohamad A A, Mojtabi A K, 2007, "Double diffusion natural convection in open lid enclosure filled with binary fluids," International Journal of Thermal Sciences, 46(2), 112-117. https://doi.org/10.1016/j.ijthermalsci.2006.04.006

Zhang H W, Tao W Q, He Y L, \& Zhang, W, 2006, "Numerical study of liquid film cooling in a rocket combustion chamber," International Journal of Heat \& Mass Transfer, 49(1), 349-358. https://doi.org/10.1016/j.ijheatmasstransfer.2005.06.017

Zhao F Y, Liu D, Tang G F, 2008, "Natural convection in a porous enclosure with a partial heating and salting element," International Journal of Thermal Sciences, 47(5), 569-583. https://doi.org/10.1016/j.ijthermalsci.2007.04.006 